\title{
Optimizing use of 5-ASA in the treatment of ulcerative colitis: Focus on patient compliance and adherence
}

This article was published in the following Dove Press journal:

Patient Related Outcome Measures

5 June 2010

Number of times this article has been viewed

\author{
Steven J Bernick' \\ Sunanda Kane ${ }^{2}$ \\ 'Department of Gastroenterology, \\ Naval Medical Center, San \\ Diego California; ${ }^{2}$ Division of \\ Gastroenterology and Hepatology, \\ Mayo Clinic, Rochester, \\ Minnesota, USA
}

\begin{abstract}
Ulcerative colitis is a chronic condition that requires long-term treatment. The first-line therapy remains 5-ASA, which is available in a variety of different formulations and dosing schedules. Multiple studies have demonstrated that adherence rates to prescribed 5-ASA products is below what would have been expected with significant consequences for important outcomes. Worse disease outcomes, higher medical costs, and even potentially higher rates of colorectal cancer have been associated with nonadherence. Nonadherence is multifactorial, fluid in nature over time, and dependent on disease activity level. Interventions to improve adherence rates have to be individualized. With the advent of simpler dosing regimens it was assumed that adherence rates would improve, but this has not necessarily been the case. Despite our current knowledge about nonadherence, it remains difficult to manage in the long term.
\end{abstract}

Keywords: ulcerative colitis, adherence, mesalamine, persistence, compliance

\section{Introduction}

Ulcerative colitis (UC), a chronic disease characterized by mucosal inflammation of the rectum and colon, is a form of inflammatory bowel disease (IBD) that is unpredictable in nature and generally requires lifelong treatment. Affecting approximately 500,000 individuals in the US with an incidence of 8-12 per 100,000 population per year, this disease presents a significant population health care burden. ${ }^{1}$ Symptoms such as bloody diarrhea, fecal urgency, tenesmus, and abdominal pain are disease hallmarks, and can lead to significant decline in an individual's quality of life. The clinical course is marked by episodes of relapse and remission frequently accompanied by disease progression over time, with an increase in the number and severity of disease-related complications. As with other chronic illnesses, a patient's ability to understand and adhere to a prescribed course of medical treatment is the fundamental principle in the successful management of their disease. Unfortunately, multiple factors often interfere with optimum patient adherence and act as barriers to the effective management of their disease.

First-line therapy for mild to moderate UC is 5-aminosalicyclic acid (5-ASA). Multiple formulations are currently available. Oral mesalamine delivered to the terminal ileum and cecum (Asacol ${ }^{\circledR}$ and Lialda ${ }^{\circledR}$ ) at doses of 1.6 to $4.8 \mathrm{~g}$ daily or throughout the gastrointestinal tract (Pentasa ${ }^{\circledR}$ ) at 2 to $4 \mathrm{~g}$ daily in patients with mildly to moderately active ulcerative colitis is effective for inducing remission, and Asacol 0.8 to $1.6 \mathrm{~g}$ daily, Pentasa $4 \mathrm{~g}$ daily, and Apriso ${ }^{\circledR} 1.5 \mathrm{~g}$ daily are effective for maintaining remission. Balsalazide and olsalazine, also 5-ASA agents, are prodrugs delivered to the colon, with indications for induction and maintenance, respectively. 5-ASA agents
Correspondence: Sunanda Kane

Division of Gastroenterology and

Hepatology, Mayo Clinic 200

First Street SW, Rochester,

MN 55905, USA

Tel +l 5072840959

Fax +I 5072840538

Email kane.sunanda@mayo.edu 
are also available in enema and suppository forms to treat the left colon and rectum directly when appropriate. Given their efficacy and favorable safety profile, the management of UC would seem to be relatively straightforward.

From the health care perspective, "adherence" is a term used to describe the extent to which patients follow the advice of health care professionals regarding their medication and disease management, and is generally interchangeable with "compliance". ${ }^{2}$ Adherence can be viewed as the cumulative combination of both compliance (medication consumption as directed) and persistence (the duration of time over which medication is consumed). Considering both compliance and persistence as separate factors becomes important in chronic illnesses such as UC, particularly given the significant variation in disease severity that generally occurs over the course of a patient's lifetime.

Adherence is generally associated with improved clinical outcomes in most medical conditions. Unfortunately, remission of disease can lull patients into a false sense of "wellness", tempting them to taper their medications or discontinue them completely. Medical recommendations may be easy to follow in the short term, but become burdensome when they are part of a lifelong strategy that requires extended vigilance. Data to corroborate this theory comes from a study of 5-ASA for UC in a cohort of patients followed at a single US institution in which only $60 \%$ of patients were able to comply with their prescribed treatment. ${ }^{3}$ However, such disappointing adherence rates are not limited to US populations. A European survey found that the nonadherence rate across Europe was nearly $30 \%$ in IBD patients. ${ }^{4}$

The clinical impact of nonadherence to therapy for UC has also been clearly associated with a greater risk of symptomatic relapse and reduced quality of life. Indeed, those patients with quiescent UC who failed to adhere to their prescribed 5-ASA regimen had a $61 \%$ chance of relapse, compared with just $11 \%$ among those who were compliant. ${ }^{3}$ A survey of 451 patients with UC suggests that some patients with UC perceive substantially more negative impact upon their lives compared with patients with other chronic illnesses. ${ }^{5}$ It is important, however, to keep in mind that these are patients with extensive disease, whereas the management principles for those with limited proctitis may not mandate chronic therapy.

The recently released results of the SIMPLE (Strategies in Maintenance for Patients Receiving Long term Therapy) trial further support what is intuitively obvious, ie, patients' compliance correlates with lower rates of disease recurrence. In this study 208 UC patients in remission were followed over a 12-month period. The mean overall compliance was $87 \%$, with those who were compliant having lower rates of disease recurrence at six and 12 months $(20.6 \%$ versus $36.1 \%$ at six months and $31.2 \%$ versus $52.5 \%$ at 12 months in compliant and noncompliant patients, respectively). ${ }^{6}$

The financial implications of nonadherence are also far reaching in the US as well as other countries. UC-related health care costs are estimated at $\$ 100$ billion in the US and 100 million in the UK annually, and UC accounts for $10 \%$ of all hospital admissions. ${ }^{7,8} \mathrm{~A}$ large health care claims database research initiative has demonstrated an association between noncompliance and increased health care costs for both out- and inpatient care in the US, and a retrospective study from the UK found that disease flare was associated with a 2-3-fold cost increase over a six-month period for those patients successfully managed as outpatients, with a more than 20-fold increase in cost if hospitalization was required. ${ }^{9,10}$

Patterns of nonadherence include under- or overconsuming medication, taking doses at inappropriate intervals, or administrating medication incorrectly (as in subcutaneous injection therapy or retention enemas). Although clinicians should be reticent to give the impression that missing doses is acceptable, we should nonetheless review this possibility with patients. As a component of ongoing education, patients should learn what to do if they miss a dose, when it is permissible to skip a dose, or modify a subsequent dose. This is especially importance in elderly patients, because they are at particular risk of inappropriate medicine consumption secondary to cognitive impairment or complicated medication regimens.

Inappropriate altering of dosing schedules may be secondary to lifestyle difficulties or inaccurate perceptions of medication delivery properties. For example, many patients find it difficult to take their medicines while at work, and younger patients feel inhibited or embarrassed about taking medicines at school. It is prudent to ask adult patients their line of work and daily schedule, so that determining whether dosing at work will prove difficult. Patients who work odd hours or multiple jobs may get confused if told to take medications before breakfast or before bed because their time schedules are not necessarily routine. In these instances, it is advisable to consider therapies that require less frequent dosing, and certainly nothing more than twice-daily dosing is required.

The incorrect administration of topical therapies can be attributed to inadequate instruction from either physician or pharmacist. Rectal administration of medication can be 
embarrassing to discuss in an open manner, and patients, rather than admit to ignorance, attempt to self-administer enemas inappropriately, potentially rendering them ineffective. Patients need to be reminded that enemas are called "retention" enemas for a reason. Living quarters are also important to keep in mind, because a patient living with others in close proximity (such as in a dormitory) is very unlikely to use enemas at all.

\section{Predictors of medication nonadherence}

A wide variety of factors can be implicated in contributing to nonadherence in patients with $\mathrm{UC}$, although the data are somewhat conflicting. In general, nonadherent patients are statistically more likely to be male (67\% versus $52 \%$ in adherent patients, respectively), of single marital status ( $86 \%$ versus $53 \%$ ), have left-sided disease as opposed to pancolitis $(83 \%$ versus $51 \%$ ), or be taking four or more concomitant medications $(60 \%$ versus $40 \%) .{ }^{11}$ Other factors, including the approach and attitude of the physician, perception of the patient, medication side effects, patient beliefs about the necessity and efficacy of the medications, prescription costs, and quiescent disease have also been linked to nonadherence. ${ }^{7,12}$ Furthermore, multiple daily doses of medication, undesirable routes of administration, poor education on the importance of maintenance therapy, full-time employment, psychological distress, younger age, new patient status, and shorter disease duration have been shown, at least in some studies, to impact negatively on patient compliance. ${ }^{2}$

A study by Kane et al showed that persistence with 5-ASA treatment in UC also varied with disease activity. Within the first three months of diagnosis, ie, a time correlated with higher levels of disease activity, a rapid decline of nearly $40 \%$ in patient refills (57\% were persistent over three months) was noted. A decline over time in the later, chronic, possibly asymptomatic phase also occurred, albeit at a slower rate. The type of 5-ASA preparation did not seem to have an effect on adherence in this study. Interesting, the authors also noted the increasing practice of mail-order prescription refills had a deleterious effect on compliance with treatment, concluding that this was related to less frequent contact with health care professionals, which has been previously shown to influence persistence with UC treatment significantly. ${ }^{13}$ Factors related to noncompliance at three months were different than those at 12 months, suggesting a dynamic process that cannot be easily predicted based on a one-time analysis. Newer work suggests, however, that those patients adherent at 12 months are also likely to be adherent at 18 months. In this analysis, at least 44,000 patients who has been given prescriptions for 5-ASA were followed for 18 months. The highest proportion of patient persistence was $13 \%$ for those using once-daily Multi-Matrix System ${ }^{\circledR}$ (MMX) mesalamine compared with 5\% rates for all other formulations. Of interest, in this study males were more likely to be persistent, as well as those aged 44-55 years. Additionally, persistence was also more likely if the agent was prescribed by an internist versus a gastroenterologist. ${ }^{14,15}$ These data would suggest that the relationship between patient and physician is indeed an important aspect to the likelihood of continued persistence, as suggested previously by Levy et al. ${ }^{12}$

\section{Patient barriers to adherence}

Patient-related factors include the degree of disease-specific education received from health care providers, patient comprehension of instructions for proper medication use (known as "health literacy"), understanding of potential consequences of nonadherence, extent of self-management skill and abilities, and strength of a support system. Only by recognizing and addressing these issues can patients be expected to maintain adherence to any medical regimen. It is important to remember that even the most "educated" patient may confuse instructions. For example, "take one tablet twice a day" could be interpreted as taking a single pill, cutting it in half, and taking each half two times a day versus taking a full tablet two times a day.

Multiple studies have examined the many barriers that serve to reduce patient adherence. Foremost among these is plain forgetfulness, which was cited as a reason for nonadherence by $50 \%$ of patients in a review by Kane in 2006. ${ }^{16}$ This form of noncompliance can be regarded as a form of illness denial, because multiple daily dosing may make patients acutely aware of their chronic illness status. When asked for other reasons for nonadherence to their prescribed regimen, $30 \%$ of patients in this study cited too many pills, while $20 \%$ stated they did not believe that they required as much medication as prescribed to them by their physician.

Another useful method for examining the barriers to patient adherence is to divide these barriers into those that are patient-related, physician-related, medication-related, and cost-related. ${ }^{2,16}$ Each of these areas offers a potential point of intervention at which patient compliance can be targeted and improved. Alternatively, in the largest systematic review of studies measuring variables associated with nonadherence to IBD medications to date, Jackson et al divided 
these variables into demographic, clinical, treatment-related, and psychosocial. ${ }^{7}$ This study challenged some previously held concepts (for example, that male gender is associated with an increased rate of nonadherence), and noted specifically that none of the frequently measured demographic, treatment-related, and clinical variables were consistently associated with nonadherence, concluding that adherence is associated with a more complex array of variables than simply those relating to the treatment regimen, and that typically recorded clinical, demographic, and treatment factors are unlikely to predict reliably which patients are nonadherent.

\section{Improving patient adherence}

In order to determine how to individualize patient care to optimize adherence, it is important that health care providers possess the proper tools to screen for nonadherence and determine those patients at highest risk for nonadherent behavior. Current work is underway using predictive tools, such as the Modified Morisky Medication Scale (MMS) and the Medication Adherence Report Scale (MARS). The MARS is a simple one-page survey tool that patients complete in the waiting room of the physician's office. ${ }^{17}$ Originally developed to predict adherence to antihypertensive medications, these tools have undergone several revisions since the 1980s, and may be useful in identifying patients who are at high risk for nonadherence, and may therefore be better suited for a visit that included discussion regarding medications. Unfortunately validation of these instruments has yielded mixed results, and none has garnered widespread support.

As mentioned, there are a variety of factors that play a role in patient adherence (Table 1). These factors can be grouped into those associated with treatment, with the disease, and with the individual. Each of these areas can be considered as a potential point for intervention in which adherence can be reinforced and, if necessary, improved.

The establishment of a therapeutic relationship between the patient and the health care provider is a key strategy in

Table I Barriers to adherence

- Lack of therapeutic relationship

- Patient forgetfulness

- Fear of side effects, adverse events

- Cost

- Lack of appropriate knowledge regarding medicine

- Comorbid conditions, such as mental health disorders improving patient compliance. This should be conceptualized as a two-way alliance whereby patients play an active role in their treatment, understand their responsibilities, and are informed on how their decisions and actions may affect the long-term course of their disease (Table 2). ${ }^{18}$ Given the unpredictable nature of $\mathrm{UC}$, it seems reasonable that any approach that places high value on patient empowerment can provide patients with a much needed sense of control over their disease. The benefits of such an approach have been demonstrated in subgroup analysis, suggesting that improvements in adherence can be realized through increased support, encouragement, and patient education. ${ }^{19}$

Also integral to a successful therapeutic relationship is the concept of "interval empathy". Demonstrations of empathy during clinical encounters, defined as the ability to understand and identify with a patient's feelings, perspective, and paradigm, have been associated with improved patient satisfaction and adherence to treatment. ${ }^{20}$ Despite this, even after years in medical practice, expression of empathy during clinical encounters is frequently lacking, as evidenced in a study of recorded encounters between oncologists and patients during which no empathetic expression was noted in over one-third of encounters with patients where it would have been appropriate. ${ }^{21} \mathrm{~A}$ more recent study showed even more dismal results, with empathy provided in only one out of every 10 instances where it would have otherwise been appropriate. ${ }^{22}$ By consciously making an effort to increase one's empathy during each patient visit, patient satisfaction with the communication process and subsequently their overall adherence may be improved.

As previously mentioned, patient education about and understanding of their disease process, treatment plan, and management goals is also important in maintaining or improving adherence. Therapeutic goals, such as increasing the chances of disease regression, decreasing the chances of disease progression, and reducing the risk for the development of colorectal cancer, should be discussed with patients early in their treatment and periodically reinforced. Providing

Table 2 A health communication model for optimizing adherence

- Provide explicit directions

- Emphasize the importance of therapy

- Supply written information combined with oral counseling

- Clarify the purpose of the medication or intervention

- Repeat important points at each visit

- Give consistent advice 
patients with evidence of the effects of medication adherence, such as improvement in biochemical indices of disease or photographic documentation of mucosal healing, can provide tangible evidence of treatment efficacy. One may even find that reviewing such demonstrable goals with patients in advance, combined with a visual aid or teaching tool that pictorially illustrates where their disease lies within the overall IBD spectrum, may be helpful.

Another aspect of patient education is the concept of self-management. Robinson et al demonstrated in a UK population that patients who were given instructions and a treatment algorithm for treating flares of their disease used fewer health care resources, had fewer flares, and a shorter duration of active symptoms for those flares that did occur. ${ }^{23}$ Another group demonstrated the improved outcomes that occurred when employing the use of a patient support program that incorporated the help of a nurse available to answer questions regarding disease and medications, even when the patient's physician was not available. ${ }^{24}$

Several studies have provided specific areas to target in attempting to ensure patient adherence. Simplification of treatment using less intrusive drug delivery systems and more convenient dosing regimens has led to improved patient adherence in the treatment of a variety of other disorders, and it seems reasonable that similar results would be found in the treatment of UC. In fact, multiple studies have shown an association between both a lower pill burden and once-daily dosing, with improved patient adherence. ${ }^{20}$

In addition, a landmark study of 1023 patients followed over a 12-month period demonstrated that once-daily dosing of delayed-release mesalamine at doses of 1.6 to $2.4 \mathrm{~g}$ /day is as effective as twice-daily dosing for maintenance of clinical remission in mild to moderate UC. ${ }^{25}$ Utilizing the MARS questionnaire, this study also noted that at 12 months patients were more satisfied with the once-daily dosing regimen, and preferred taking medication fewer times each day. Further supporting the use of once-daily dosing, emerging data have demonstrated a greater remission rate at one year (73.8\% versus $63.6 \%$ when using $2 \mathrm{~g}$ once daily versus $1 \mathrm{~g}$ mesalamine twice daily), with patient questionnaires showing significantly greater compliance and acceptability with the once-daily dosing schedule. ${ }^{26}$ It is worth noting that several studies have demonstrated the cost-effectiveness of 5-ASA therapy in the treatment of UC, and this becomes an important aspect in assuring quality of care. ${ }^{27,28}$

Consideration may also be given to trading improved efficacy, such as that seen with 5-ASA enemas when compared with oral formulations for left-sided UC, for improved adherence to an oral regimen. In a small pilot study of patients in this category, the overall rate of adherence to oral 5-ASA treatment was $97 \%$ versus $87.5 \%$ with rectal 5-ASA. ${ }^{29}$ However, it must be noted that a change to once-daily dosing does carry the potential for an actual decrease in adherence if the patient misses their scheduled dose, because the patient will then miss 24 hours of therapy instead of just 8-12 hours had they been taking two or three doses daily. Interestingly, however, a survey of 100 Canadian patients with UC revealed that factors such as medication efficacy and safety were more important than those related to dosing regimen or cost. In this survey, speed of symptom relief and fewer side effects were rated as the most important factors when considering a UC medication. ${ }^{30}$

As previously discussed, routinely questioning patients about their adherence has been suggested. This can be accomplished not only by a simple interview during the patient intake process, routine pill checks, or use of managed care databases, where available. There is also a medication adherence scale available and already validated in hypertension, another chronic condition characterized by long periods without symptoms. ${ }^{31}$ Research is currently underway to validate this tool for UC patients. Each of these methods can provide the opportunity to open a dialog between the physician and patient regarding adherence, allowing for patient education and identifying any potential barriers that may be interfering with the patient's ability to comply with their treatment regimen.

Psychologic literature points to using a quick acronymbased strategy for addressing compliance issues, known as "COPE". This strategy captures the essence of many of the above concepts. First, Communicate with your patient about the importance of medication adherence at every visit. Establish and nurture a therapeutic alliance based upon a respect for the patient's beliefs and wishes, without diminishing those of the physician, so that both the physician and patient are able to proceed on the basis of reality, and not on misunderstanding, distrust, or concealment. ${ }^{32}$ Next, Obtain your patient's commitment to therapeutic objectives. Furthermore, one should Promote emotional and psychological support, utilizing the concept of "interval empathy". Finally, Educate the patient and family about their disease process, treatment plan, and management goals. A wide variety of educational materials are available for this purpose, and should be utilized where appropriate (Table 3). 
Table 3 Techniques for maximizing adherence in UC patients

- Use allied health staff to medication history

- Simplify regimen when possible

- Use clinic appointments to discuss barriers to adherence

- Explain consequences of nonadherence (cost, complications, disease activity)

- Negotiate with patients to maximize medication exposure

\section{Conclusion}

Patient adherence to their treatment regimen is important in the management of chronic diseases such as UC. A number of factors can negatively impact patient adherence, with deleterious consequences in both the short and long term. The development of a therapeutic alliance, use of "interval empathy", and education of the patient and their family are paramount to ensuring adherence. Specific interventions, such as simplification of treatment dosing schedules, eg, changing to once-daily mesalamine, altering routes of medication administration, and routinely querying the patient regarding adherence, are easy management tools that may prove beneficial. Finally, the COPE acronym captures the essence of many important strategies useful in reinforcing and achieving patient compliance. Not unlike the previous trials with self-management protocols, proposed research includes the use of home telemanagement to improve adherence, decrease cost, and increase quality of life, but will be expensive and time-consuming.

\section{Disclosure}

SJB has no conflicts to disclose. SK serves as a Consultant to Kyorin, Shire, and Warner Chilcott, and receives research support from Shire and Warner Chilcott.

\section{References}

1. Kornbluth A, Sachar D. and the Practice Parameters Committee of the American College of Gastroenterology. Ulcerative colitis practice guidelines in adults: American College of Gastroenterology Practice Parameters Committee. Am J Gastroenterol. 2010;105:501-523.

2. Hawthorne AB, Rubin G, Ghosh S. Medication non-adherence in ulcerative colitis - strategies to improve adherence with mesalamine and other maintenance therapies. Aliment Pharmacol Ther. 2008;27:1157-1166.

3. Kane S, Huo D, Aikens J, Hanauer S. Medication nonadherence and the outcomes of patients with quiescent ulcerative colitis. Am J Gastroenterol. 2003;114:39-44.

4. Robinson, A. Review article: Improving adherence to medication in patients with inflammatory bowel disease. Aliment Pharmacol Ther. 2008;27 Suppl 1:9-14.

5. Rubin DT, Siegel CA, Kane SV. The impact of ulcerative colitis from patients' and physicians' perspective: Results from the UC: NORMAL survey. Inflamm Bowel Dis. 2009;15:581-588.

6. Kane S, Solomon D, Palmen M, Barrett K. Association between compliance and clinical recurrence in patients with quiescent ulcerative colitis receiving $\mathrm{MMX}^{\circledR}$ mesalamine $2.4 \mathrm{~g} /$ day as maintenance therapy: Results of the Phase IV SIMPLE trial. Gastroenterol. 2010;136 Suppl 1: Abstr. 765.
7. Jackson CA, Clatworthy J, Robinson A, Horne R. Factors associated with adherence to oral medication for inflammatory bowel disease: A systemic review. Am J Gastroenterol. 2010;105:535-539.

8. Lakatos P. Prevalence, predictors, and clinical consequences of medical adherence in IBD: How to improve it. World J Gastroenterol. 2009;15: 4234-4239.

9. Kane S, Shaya F. Medication non-adherence is associated with increased medical health care costs. Dig Dis Sci. 2008;53(4):1020-1024.

10. Bassi A, Dodd S, Williamson P, et al. Cost of illness of inflammatory bowel disease in the UK: A single centre retrospective study. Gut. 2004;53:1471-1478.

11. Kane SV, Cohen RD, Aikens JEB. Predictors of non-compliance with mesalamine in quiescent ulcerative colitis. Am J Gastroenterol. 2001;96:2929-2932.

12. Levy RL, Feld AD. Increasing patient adherence to gastroenterology treatment and prevention regimens. Am J Gastroenterol. 1999;94: $1733-1742$.

13. Kane S, Accortt N, Magowan S, Brixner D. Predictors of persistence with 5-aminosalicylic acid therapy for ulcerative colitis. Aliment Pharmacol Ther. 2009;9:855-862.

14. Kane S, Sumner M, Solomon D, Jenkins M. Continuing persistence with mesalamine therapy: Results from patients persistent with long-term therapy. Am J Gastroenterol. 2009;104 Suppl 3:A1271.

15. Kane S, Sumner M, Solomon D, Jenkins M. Factors affecting persistence with mesalamine therapy: Results from a large pharmacy database. $\mathrm{Am}$ J Gastroenterol. 2009;104 Suppl 3:A1272.

16. Kane SV. Systematic review: Adherence issues in the treatment of ulcerative colitis. Aliment Pharmacol Ther. 2006;23:577-585.

17. Morisky DE, Alfonso A, Krousel-Wood M, et al. Predicitve validity of a medication adherence measure in an outpatient setting. $J$ Clin Hypertens. 2008;10:348-354.

18. Prochaska JO, DiClemente CC. Stages of change in the modification of problem behaviors. Prog Behav Modif. 1992;28:183-218.

19. Tindall W, Boltri J, Wilhelp S. Mild-to-moderate ulcerative colitis: Your role in patient compliance and health care costs. JMPC. 2007;13:S1-S12.

20. Stewart MA. What is a successful doctor patient interview? A study of interactions and outcomes. Soc Sci Med. 1984;19:167-175.

21. Fallowfield L, Jenkins V, Farewell V, et al. Efficacy of a communication skills training model for oncologists: A randomized controlled trial. Lancet. 2002;359:650-656.

22. Morse DS, Edwardsen EA, Gordon HS. Missed opportunities for interval empathy in lung cancer communication. Arch Intern Med. 2008;168:1853-1858.

23. Robinson A, Thompson DG, Wilkin D, et al. Guided self-management and patient-directed follow-up of ulcerative colitis: A randomized trial. Lancet. 2001;358(9268):976-981.

24. Tukey M, Flachuk K, Cheifetz A, Moss A. A patient support program (PSP) to enhance medication adherence and quality-of-life in patients prescribed mesalamine for ulcerative colitis - a pilot study. Am J Gastroenterol. 2008;73 Suppl:A288.

25. Sandborn W, Korzenik J, Lashner B, Leighton J, et al. Once-daily dosing of delayed-release oral mesalamine (400-mg tablet) is as effective as twice-daily dosing for maintenance of remission in ulcerative colitis. Gastroenterology. 2010;138:1286-1296.

26. Dignass A, Bokemeyer B, Ademek H, et al. Mesalamine once daily is more effective than twice daily in patients with quiescent ulcerative colitis. Clin Gastroenterol Hepatol. 2009;7:762-769.

27. Yen EF, Kane SV, Ladabaum U. Cost-effectiveness of 5-aminosalicylic acid therapy for maintenance of remission in ulcerative colitis. Am J Gastroenterol. 2008;103:3094-3105.

28. Higgins PD, Rubin DT, Kaulback K, Schoenfeld PS, Kane SV. Systematic review: Impact of non-adherence to 5-ASA products on the frequency and cost of ulcerative colitis flares. Aliment Pharmacol Ther. 2009;29(3):247-257. 
29. Prantera C, Viscido A, Biancone L, et al. A new oral delivery system for 5-ASA: Preliminary clinical findings for MMX. Inflamm Bowel Dis. 2005;11:421-427.

30. Gray J, Leung E, Scales J. Treatment of ulcerative colitis from the patient's perspective: A survey of preferences and satisfaction with therapy. Aliment Pharmacol Ther. 2009;29:1114-1120.
31. Krousel-Wood M, Islam T, Webber LS, Re RN, Morisky DE, Muntner P. New medication adherence scale versus pharmacy fill rates in seniors with hypertension. Am J Manag Care. 2009;15(1):59-66.

32. Scherman MH, Lowhagen O. Drug compliance and identity: Reasons for non-compliance. Experiences of medication from persons with asthma/allergy. Patient Educ Counsel. 2004;54:3-9.

Patient Related Outcome Measures

\section{Publish your work in this journal}

Patient Related Outcome Measures is an international, peer-reviewed, open access journal focusing on treatment outcomes specifically relevant to patients. All aspects of patient care are addressed within the journal and practitioners from all disciplines are invited to submit their work as well as healthcare researchers and patient support groups. Areas covered will include: Quality of life scores; Patient satisfaction audits; Treatment outcomes that focus on the patient; Research into improving patient outcomes; Hypotheses of interventions to improve outcomes; Short communications that illustrate improved outcomes; Case reports or series that show an improved patient experience; Patient journey descriptions or research.

Submit your manuscript here: http://www.dovepress.com/patient-related-outcome-measures-journal 